\title{
CLONING THE ENTANGLEMENT OF A PAIR OF $d$-DIMENSIONAL QUANTUM SYSTEMS
}

\author{
E. Karpov ${ }^{a}$, P. Navez ${ }^{a, b}$, N.J. Cerf ${ }^{a}$ \\ ${ }^{a}$ Quantum Information and Communication, École Polytechnique, Université Libre de Bruxelles, \\ Brussels, Belgium \\ ${ }^{b}$ Labo Vaste-Stoffysica en Magnetisme, Katholieke Universiteit Leuven, Heverlee, Belgium
}

\begin{abstract}
We derive a quantum cloning machine that maximizes the entanglement of formation of the two copies of any maximally entangled input state, while preserving the separability of all unentangled input states. In addition, it is proven to optimally duplicate the entanglement of formation of all isotropic input states. For large $d$, the cloning machine behaves classically and outperforms a local entanglement cloner, studied for comparison.
\end{abstract}

Мы рассчитали квантовую копирующую машину, которая обеспечивает максимальное перепутывание (entanglement of formation) в обеих копиях максимально перепутанного исходного квантового состояния, при этом копии неперепутанных состояний сохраняются неперепутанными. Кроме того, мы доказали, что перепутанность всех изотропных состояний копируется нашей копирующей машиной оптимальным образом. Наша копирующая машина превосходит локальную копирующую машину, которую мы изучали для сравнения, а при большой размерности пространства Гильберта она функционирует как классический переключатель.

\section{INTRODUCTION}

Quantum entanglement is an important resource for quantum communication, quantum computation, and quantum cryptography. Therefore, it is important to know to what extent this resource is reproduced when entangled states are duplicated. A perfect cloning of arbitrary quantum states is forbidden by the no-cloning theorem [2]. However, an imperfect cloning is possible and various quantum cloning machines (QCM), which duplicate quantum states with the highest fidelity, have been proposed following the seminal paper of Buzek and Hillery [3]. However, the question of whether quantum entanglement can be cloned or not has been raised only recently, and a QCM was proposed that optimally (but imperfectly) clones the entanglement of entangled pairs of two-dimensional systems (qubits) while preserving separability [4]. Separability preservation is an important feature of QCM following an entanglement no-cloning principle that was formulated in [4]: «if a quantum operation can be found that perfectly duplicates the entanglement of all maximally entangled (ME) states, then it does not necessarily preserve separability». It means that such a «perfect» QCM produces entangled clones of separable states, i.e. creates undesired entanglement.

In this conference paper, we report on the extension of these results to $d$-dimensional quantum systems, presented in the paper [1]. In Sec. 1, we define a (symmetric) cloning 
transformation acting on $d \times d$-dimensional quantum states and determine the parameters of this transformation that maximize the amount of entanglement of the two clones of any ME state. In Sec. 2, we compare our optimal $d \times d$ entanglement cloner to a real and universal cloning machine, as well as to a «local» cloning transformation that is achieved by applying a separate universal cloning machine to each component of the bipartite system. In Sec. 3, we analyze the entanglement of the clones in terms of fidelity and show that this actually leads to maximizing the entanglement of formation of the clones provided that we restrict ourselves to isotropic input states (including ME states) and cloning machines that are covariant under local unitaries. In Sec. 4 we check that our optimized cloning transformation produces separable clones in the case of unentangled input states. Finally, we present our conclusions.

\section{OPTIMAL $d \times d$ ENTANGLEMENT CLONER}

Following the ideas presented in [4], we seek for a cloning transformation that (i) preserves separability, and (ii) maximizes the entanglement of the two clones resulting from any ME input state. We shall characterize a cloner by considering the transformation of an input that is maximally entangled with a reference system (see [5]). Using the isomorphism between completely positive (CP) maps $\mathcal{S}$ and positive semidefinite operators $S \geqslant 0$ on the tensor product of input and output Hilbert spaces $\mathcal{H}_{\text {in }} \otimes \mathcal{H}_{\text {out }}[6]$, we shall represent our cloning transformation by a quantum state $S$.

Let us illustrate this isomorphism following Ref. [7]. Consider first ME state on $\mathcal{H}_{\text {in }} \otimes$ $\mathcal{H}_{\text {out }}=\mathcal{H}^{\otimes 2}$

$$
\left|\Phi^{+}\right\rangle=\frac{1}{\sqrt{d}} \sum_{j=0}^{d-1}|j\rangle_{1}|j\rangle_{2},
$$

where $d=\operatorname{dim}\left(\mathcal{H}_{\text {in }}\right)$. Applying the map $\mathcal{S}$ to subsystem 2 one obtains the resulting (generally mixed) quantum state - linear positive operator

$$
S=I_{1} \otimes \mathcal{S}_{2}\left(d\left|\Phi^{+}\right\rangle\left\langle\Phi^{+}\right|\right)
$$

that is isomorphic to $\mathcal{S}$ (see Fig. 1). A trace preserving map satisfies

$$
\operatorname{Tr}_{\text {out }}[S]=\mathbb{1}_{\text {in }} .
$$

The CP map $\rho_{\text {out }}=\mathcal{S}\left(\rho_{\text {in }}\right)$ can be expressed in terms of $S$ as follows:

$$
\rho_{\text {out }}=\operatorname{Tr}_{\text {in }}\left[\rho_{\text {in }}^{T} \otimes \mathbb{1}_{\text {out }} S\right],
$$

where $T$ denotes the transposition in the Schmidt basis of $\left|\Phi^{+}\right\rangle$. We shall call «reference» the part of state $S$, which corresponds to $\mathcal{H}_{\text {in }}$ and denotes this Hilbert space as $\mathcal{H}_{\mathcal{R}}$.

For the $1 \rightarrow 2$ cloning transformation first we observe that the output Hilbert space $\mathcal{H}_{\text {out }}$ must include at least a tensor product $\mathcal{H}_{a} \otimes \mathcal{H}_{b}$ of two clones «a» and «b». In addition, one needs to introduce an ancilla that we shall denote as $\mathcal{A}$. Therefore, the total output state must be endowed with the following tensor product structure $\mathcal{H}_{\text {out }}=\mathcal{H}_{a} \otimes \mathcal{H}_{b} \otimes \mathcal{H}_{\mathcal{A}}$. The ancilla is necessary in order to purify the state $S$ into a pure state $|S\rangle$ such that $S=\operatorname{Tr}_{\mathcal{A}}[|S\rangle\langle S|]$ [5]. Together with the reference component the overall state $|S\rangle$ belongs to the product space 


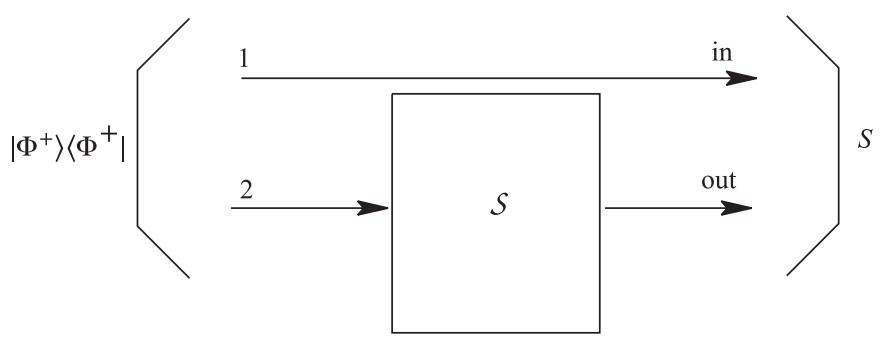

Fig. 1. Isomorphism between a $\mathrm{CP}$ map $\mathcal{S}$ and a linear positive operator $S$

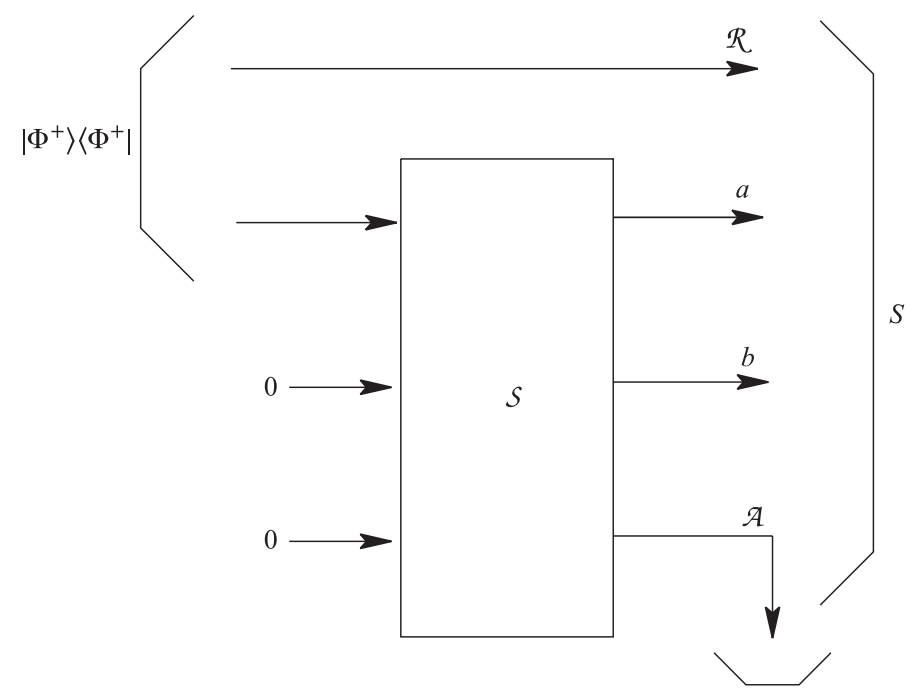

Fig. 2. Cloning transformation as a $\mathrm{CP}$ map $\mathcal{S}$ or as a state $S$

$\mathcal{H}_{\mathcal{R}} \otimes \mathcal{H}_{a} \otimes \mathcal{H}_{b} \otimes \mathcal{H}_{\mathcal{A}}$ (see Fig. 2). Thus, following the arguments of Refs. [5] and [7], we shall construct the state $|S\rangle$ as a linear combination of products of four components, namely, the reference system, which we denote as $\mathcal{R}$, two blank systems for future clones denoted as $a$ and $b$ and an ancilla denoted by $\mathcal{A}$. As we are interested in cloning entanglement, all four components are now $d^{2}$-dimensional bipartite states. The general form for such a cloning transformation is defined in the computational basis $\{|i\rangle\}$ (where $|i\rangle=\left|i_{A}\right\rangle\left|i_{B}\right\rangle$ ) by the state

$$
|S\rangle_{\mathcal{R}, a, b, \mathcal{A}}=\sum_{i, j, k, l} s_{i j k l}|i\rangle_{\mathcal{R}}|j\rangle_{a}|k\rangle_{b}|l\rangle_{\mathcal{A}}
$$

All the summations here are $d^{2}$-dimensional since each index $i, j, k$, or $l$ actually represents a couple of indices running each from 0 to $d-1$, e.g., $i=\left\{i_{A}, i_{B}\right\}$, with $i_{A}, i_{B} \in[0 ; d-1]$. Of course, the index $A$ stands for Alice's component of the bipartite states, while $B$ stands for Bob's component.

As mentioned above, the joint state of the two clones and the ancilla is obtained by performing an appropriate projection on the reference system. Thus, for an input state 
$|\Phi\rangle=\sum_{i} n_{i}|i\rangle$, the result of the cloning transformation is of the form

$$
|\chi\rangle={ }_{\mathcal{R}}\left\langle\Phi^{*} \mid S\right\rangle_{\mathcal{R}, a, b, \mathcal{A}}=\sum_{i, j, k, l} s_{i j k l} n_{i}|j\rangle_{a}|k\rangle_{b}|l\rangle_{\mathcal{A}}
$$

Then, the state of any of the clones is further obtained by tracing out the ancilla and the other clone. This is a kind of global transformation that clones jointly the components $A$ and $B$ of the entangled initial state resulting in two bipartite entangled states as shown in Fig. 3, $a$.

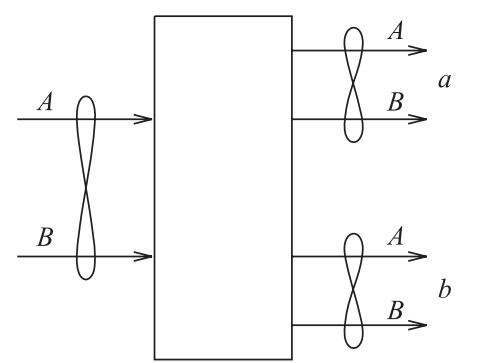

a)

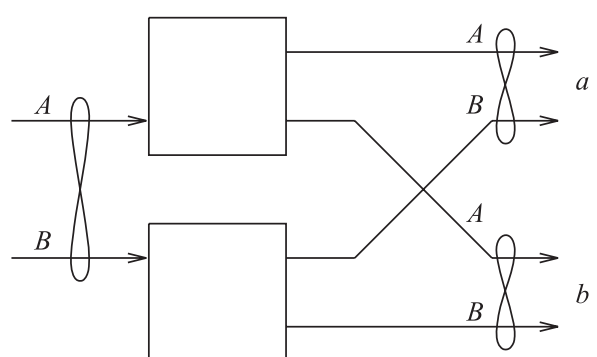

b)

Fig. 3. a) Optimal $d \times d$ entanglement cloner; $b$ ) «local» entanglement cloner, as defined in Sec. 2 . Here, $A$ and $B$ stand for Alice's and Bob's part of the bipartite state, while $a$ and $b$ stand for two clones. Entanglement is indicated by double loops

Next, we impose the following covariance condition on our cloning machine. Since we know that any local unitary operation acting on the $A$ and $B$ components of a bipartite state preserves its entanglement, we require that any of such transformations acts similarly on the clones. This condition amounts to imposing

$$
|S\rangle_{\mathcal{R}, a, b, \mathcal{A}}=U^{*} \otimes U \otimes U \otimes U^{*}|S\rangle_{\mathcal{R}, a, b, \mathcal{A}}
$$

where $U$ is the product of any two unitary transformations acting separately on each $d$ dimensional component of the bipartite state, that is

$$
U=U_{A} \otimes U_{B},
$$

where the indices $A$ and $B$ denote Alice's and Bob's components. Defined in this way, the operator $U$ possesses a $S U(d) \otimes S U(d)$ symmetry. The covariance property implies that $s_{i j k l}$ in (1) satisfies the transformation:

$$
s_{i j k l}=U_{i i^{\prime}}^{*} U_{j j^{\prime}} U_{k k^{\prime}} U_{l l^{\prime}}^{*} s_{i^{\prime} j^{\prime} k^{\prime} l^{\prime}},
$$

where $U^{*}$ denotes the matrix element-wise complex conjugate of $U$ with respect to the computational basis. (Here, the summation over all repeated indices is implicit.) If the transformation with respect to Alice's component is real, i.e. a rotation under $S O(d)$, then Eq. (5) implies that $s_{i j k l}$ is a rank-4 isotropic tensor, i.e. invariant under all possible real rotations of the reference frame. There are only three independent components of such 
an isotropic tensor (see [8]): $\delta_{i_{A} j_{A}} \delta_{k_{A} l_{A}}, \delta_{i_{A} k_{A}} \delta_{j_{A} l_{A}}$ and $\delta_{i_{A} l_{A}} \delta_{j_{A} k_{A}}$. Furthermore, if the transformation is complex, i.e. a rotation under $S U(d)$, then the term $\delta_{i_{A} l_{A}} \delta_{j_{A} k_{A}}$ must be excluded since, for example, it does not fulfil Eq. (9) with the phase transformation $U_{i j}=\mathrm{e}^{i \theta} \delta_{i_{A} j_{A}} \delta_{i_{B} j_{B}}$ for any angle $\theta$. The same reasoning holds for Bob's component. Then, combining all possible terms compatible with (9), we obtain the general form of the resulting tensor as

$$
\begin{aligned}
s_{i j k l}=A \delta_{i_{A} j_{A}} \delta_{k_{A} l_{A}} \delta_{i_{B} j_{B}} \delta_{k_{B} l_{B}}+B \delta_{i_{A} j_{A}} \delta_{k_{A} l_{A}} \delta_{i_{B} k_{B}} \delta_{j_{B} l_{B}}+ \\
+C \delta_{i_{A} k_{A}} \delta_{j_{A} l_{A}} \delta_{i_{B} k_{B}} \delta_{j_{B} l_{B}}+D \delta_{i_{A} k_{A}} \delta_{j_{A} l_{A}} \delta_{i_{B} j_{B}} \delta_{k_{B} l_{B}} .
\end{aligned}
$$

For a symmetric cloner, the output state must be invariant under the interchange of the two clones, i.e. under permutations $\left(j_{A}, j_{B}\right) \leftrightarrow\left(k_{A}, k_{B}\right)$. This implies that $A=C$ and $B=D$, so we are left with only two complex parameters $A$ and $B$ to be determined.

The covariance condition (7) guarantees that our QCM transforms all states which are equivalent to local unitaries (which have therefore the same entanglement) into equally entangled clones. In particular, the clones of all ME states will be equally entangled. Then, a cloner that is optimized on a particular ME input state will be optimal for all ME states. We choose as an initial $d \times d$ ME state

$$
|\Phi\rangle=\sum_{i_{A}, i_{B}=0}^{d-1} n_{i_{A} i_{B}}\left|i_{A}\right\rangle\left|i_{B}\right\rangle,
$$

where $n_{i_{A}, i_{B}}=\delta_{i_{A} i_{B}} / \sqrt{d}$. As we shall show later, we can maximize the entanglement of the clones simply by optimizing our QCM in terms of the fidelity of the clones,

$$
F=\left\langle\Phi\left|\rho_{a}\right| \Phi\right\rangle,
$$

where

$$
\rho_{a}=\operatorname{Tr}_{\mathcal{A}, b}[|\chi\rangle\langle\chi|]
$$

is the state of clone $a$. For the ME state (11), this fidelity is found to be

$$
F=\left(d^{2}+3\right)|A|^{2}+4|B|^{2}+\frac{4}{d}\left(d^{2}+1\right) \operatorname{Re}\left(A^{*} B\right) .
$$

Taking into account the normalization condition for the joint output state $|\chi\rangle$,

$$
2\left(d^{2}+1\right)\left(|A|^{2}+|B|^{2}\right)+8 d \operatorname{Re}\left(A^{*} B\right)=1,
$$

we can maximize the fidelity, Eq. (14), as follows. Introducing new notations

$$
\begin{cases}2\left(d^{2}+1\right) & =\alpha+\beta \\ 4 d & =\alpha-\beta\end{cases}
$$

we rewrite Eq. (15) in the form

$$
\alpha|A-B|^{2}+\beta|A+B|^{2}=1 .
$$


Then denoting

$$
\left\{\begin{array}{l}
A-B=C \\
A+B=D
\end{array}\right.
$$

and taking into account Eq. (16) we rewrite Eq. (14) as

$$
F=\frac{1}{4}\left(\alpha|C|^{2} d_{1}+\beta|D|^{2} d_{2}+2|C||D| \sqrt{\alpha \beta} \cos \left(\varphi_{D}-\varphi_{C}\right)\right),
$$

where $\varphi_{C}, \varphi_{D}$ are the arguments of the complex variables $C$ and $D$, and

$$
d_{1}=1-\frac{2(d-2)}{d(d-1)}, \quad d_{2}=1+\frac{2(d+2)}{d(d+1)} .
$$

Observing that in Eq. (19) all terms except for the cosine factor are positive and the argument of the cosine is independent of all other parameters, we conclude that $F$ is maximized when $\cos \left(\varphi_{D}-\varphi_{C}\right)=1$. Then, with the help of Eqs. (17) and (18) we can denote

$$
\cos \theta=\alpha^{1 / 2} C^{2}, \quad \sin \theta=\beta^{1 / 2} D^{2}
$$

and from Eq. (19) we obtain $F$ optimized in terms of the arguments $\varphi_{C}$ and $\varphi_{D}$ as

$$
F=\frac{1}{4}\left(d_{1} \cos ^{2} \theta+d_{2} \sin ^{2} \theta+2 \sin \theta \cos \theta\right) .
$$

After some algebra, we rewrite Eq. (22) in the form

$$
F=\frac{1}{4}\left(\frac{d_{1}+d_{2}}{2}+r \cos (2 \theta-\Delta)\right)
$$

where

$$
r=\sqrt{1+\left(\frac{d_{1}-d_{2}}{2}\right)^{2}}, \quad \sin \Delta=\frac{1}{r} .
$$

Again, observing that $\theta$ in Eq. (23) is independent of $d_{1}$ and $d_{2}$, we conclude that the maximum of $F$ is achieved when $\cos (2 \theta-\Delta)=1$, which yields

$$
F=\frac{1}{4}\left(\frac{d^{2}+1}{d^{2}-1}+\sqrt{1+\frac{4}{d^{2}}\left(\frac{d^{2}-2}{d^{2}-1}\right)^{2}}\right) .
$$

Note that, for $d=2$, this result coincides with the maximal fidelity of the entanglement cloner for two qubits obtained in [4], namely

$$
F=\frac{5+\sqrt{13}}{12} \approx 0.7171
$$

The parameters of the tensor $s_{i j k l}$ (10) maximizing $F$ in $d$ dimensions are found to be

$$
\begin{aligned}
& A=\frac{d \sqrt{1+Y(d)}-\sqrt{1-Y(d)}}{2\left(d^{2}-1\right)}, \\
& B=-\frac{d \sqrt{1-Y(d)}+\sqrt{1+Y(d)}}{2\left(d^{2}-1\right)},
\end{aligned}
$$


where

$$
Y(d)=\left(1-\frac{\left(d^{2}-2\right)^{2}}{d^{2}\left(d^{2}-1\right)^{2}+4\left(d^{2}-2\right)^{2}}\right)^{1 / 2}
$$

\section{COMPARISON WITH OTHER CLONERS}

We compare the fidelity achieved by our optimal $d \times d$-dimensional entanglement cloner, Eq. (25), with that of the universal cloner [5]

$$
F_{u}=\frac{1}{2}+\frac{1}{d^{2}+1},
$$

as well as that of the optimal real cloner [9]

$$
F_{r}=\frac{1}{2}+\frac{\sqrt{d^{4}+4 d^{2}+20}-d^{2}+2}{4\left(d^{2}+2\right)} .
$$

In order to make this comparison consistent, we have obtained formulae (30) and (31) by replacing the argument $d$ by $d^{2}$ in the original formulae. This is done because, in our consideration, the dimension $d$ stands for the dimension of each component of the bipartite input state, so that the total dimension of our input state is $d^{2}$.

In Table, we compare the fidelity $F$ of our entanglement cloner with $F_{u}$ and $F_{r}$, for several values of the dimension $d$. Our cloner performs better than the universal cloner in $d^{2}$ dimensions for all $d$, which is obviously due to the fact that the ME states span only a subset of the entire set of $d^{2}$-dimensional states. In contrast, the real $d^{2}$-dimensional cloners outperform our cloners, except if $d=2$ where they coincide [4]. This can be interpreted by noting that the set of $d^{2}$-dimensional real states is generated by $S O\left(d^{2}\right)$, with $\left(d^{2}\left(d^{2}+1\right) / 2\right)-1$ real degrees of freedom, while the set of ME states is generated by $S U(d) \times S U(d)$, with $\left(d^{2}-1\right)^{2}$ real degrees of freedom. For $d=2$ they coincide, so that our cloner provides the same fidelity as that of the real cloner in dimension 4, namely Eq. (26). This is related to the fact that the set of ME 2-qubit states is isomorphic to the set of 4-dimensional real states [4]. For $d>2$ the set of ME states is in some sense «larger» than the set of real states, so that the achievable fidelity of the entanglement cloner is lower. The fidelity of our cloner drops faster than that of the real cloner with increasing $d$, but always remains higher than the fidelity of the universal cloner. As expected, in the limit $d \rightarrow \infty$, all three fidelities tend to the asymptotic value $1 / 2$. In this limit, all quantum cloners can be interpreted simply as a classical transformation that maps the original state to one of the clones, chosen at random, the other clone being prepared in a maximally mixed state.

Interestingly, we may also compare Eq. (25) to the fidelity of a «local cloner» obtained by applying a cloner separately to Alice's and Bob's components (see Fig. 3,b). Since the state of Alice's or Bob's subsystem is maximally mixed (hence non-polarized) when the bipartite state is $\mathrm{ME}$, it is natural to use a universal $d$-dimensional cloner. We may observe that if we consider a cloning transformation that performs such a local universal cloning, then it is represented by a joint state of the same type as Eq. (5), see [5]. The only difference is that in the expression (10) for the tensor $s_{i j k l}$ all coefficients must be equal, i.e. $A=B=C=D$. 
Optimal fidelity $F$ of the $d \times d$ entanglement cloner as compared to the fidelity of the real cloner $F_{r}$ and universal cloner $F_{u}$, both in $d^{2}$ dimensions, to the fidelity of the $d \times d$ «local» cloner $F_{\text {loc }}$, and to the fidelity of a «local» cloner «improved» by classical communication $F_{\mathrm{LOCC}}$. The fidelities are shown in decreasing order

\begin{tabular}{|c|c|c|c|c|c|}
\hline$d \times d$ & $F_{r}$ & $F$ & $F_{u}$ & $F_{\text {LOCC }}$ & $F_{\text {loc }}$ \\
\hline $2 \times 2$ & 0.7171 & 0.7171 & 0.7000 & 0.625 & 0.5833 \\
$3 \times 3$ & 0.6069 & 0.6019 & 0.6000 & & 0.4583 \\
$4 \times 4$ & 0.5617 & 0.5592 & 0.5588 & & 0.4000 \\
$5 \times 5$ & 0.5398 & 0.5386 & 0.5385 & & 0.3667 \\
$6 \times 6$ & 0.5277 & 0.5271 & 0.5270 & & 0.3452 \\
\hline
\end{tabular}

Then the normalization condition (15) gives immediately $A=1 /(2(d+1))$. The substitution of this expression into Eq. (14) results in the fidelity for the local cloner:

$$
F_{\mathrm{loc}}=\frac{1}{4}+\frac{d+2}{2 d(d+1)} .
$$

This fidelity is compared in Table with that of the other cloners. It appears that cloning Alice's and Bob's parts locally leads to a much lower fidelity. Note that for $d=2$, the value of the fidelity $F_{\text {loc }}$ in Table coincides with the value $7 / 12$ obtained in [4]. In the limit $d \rightarrow \infty$, this fidelity tends to $1 / 4$, which can be easily understood as follows. To contribute to the fidelity, both cloners indeed need to map Alice's and Bob's components of the original state onto the right clone, which only happens with probability $(1 / 2)^{2}=1 / 4$. An interesting observation made in Ref. [10] is that classical communication can improve local cloners. The result for $d=2$ presented in Table shows indeed the increased value of $F_{\text {LOCC }}$ compared to the fidelity $F_{\text {loc }}$ of the local universal cloner. This value, however, is lower than the result for all considered non-local cloners.

\section{ENTANGLEMENT OF FORMATION}

In order to investigate the entanglement properties of our cloning transformation, we shall use as an entanglement measure for the clones the entanglement of formation [11], which was computed for several classes of states that are invariant under some groups of local symmetries [12]. In particular, we shall be interested in the class of states that are invariant under the transformations $U \otimes U^{*}$ for all $U \in S U(d)$, called isotropic states in [12,13]. These states may be written in a general form as [14]

$$
\rho=\frac{1-F}{d^{2}-1}(\mathbb{1}-|\Phi\rangle\langle\Phi|)+F|\Phi\rangle\langle\Phi|
$$

where $\mathbb{1}$ is the identity and $|\Phi\rangle$ is given by Eq. (11). Due to the covariance condition (7), our QCM transforms $U \otimes U^{*}$ invariant states into the states that are also invariant under $U \otimes U^{*}$. We can check that, by cloning the particular ME state $|\Phi\rangle$, which is $U \otimes U^{*}$ invariant, we 
obtain a clone of the form

$$
\begin{aligned}
\rho_{a}=\left(\left(d^{2}+2\right)|A|^{2}+2|B|^{2}+4 d \operatorname{Re}\left(A^{*} B\right)\right) \mid & |\Phi\rangle\langle\Phi|+ \\
+ & \left(|A|^{2}+2|B|^{2}+\frac{4}{d} \operatorname{Re}\left(A^{*} B\right)\right) \mathbb{1},
\end{aligned}
$$

which is indeed an isotropic state and is consistent with Eq. (14). Hence, as a consequence of our covariance condition, all ME states, which can be obtained from $|\Phi\rangle$ by applying local unitaries, are cloned onto isotropic states. For this class of states, the entanglement of formation is known for arbitrary dimensions $[12,14]$

$$
E_{F}(\rho)= \begin{cases}0, & F \leqslant \frac{1}{d}, \\ R_{1, d-1}(F), & F \in\left[\frac{1}{d}, \frac{4(d-1)}{d^{2}}\right], \\ \frac{d \log _{2}(d-1)}{d-2}(F-1)+\log _{2} d, & F \in\left[\frac{4(d-1)}{d^{2}}, 1\right],\end{cases}
$$

where

$$
\begin{aligned}
R_{1, d-1}(F) & =H_{2}(\gamma(F))+[1-\gamma(F)] \log _{2}(d-1), \\
\gamma(F) & =\frac{1}{d}[\sqrt{F}+\sqrt{(d-1)(1-F)}]^{2}, \\
H_{2}(p) & =-p \log _{2}(p)-(1-p) \log _{2}(1-p) .
\end{aligned}
$$

As shown in Fig. 4, the entanglement of formation $E_{F}(\rho)$ is a monotonically increasing function of the fidelity $F$ for isotropic states in any dimension $d$. Therefore, by optimizing our QCM in terms of fidelity we maximize, at the same time, the entanglement of the clones measured by their entanglement of formation. The circles in Fig. 4 correspond to the maximal fidelity $F$ that is achieved by our entanglement cloner, Eq. (25). They show as well the corresponding entanglement of formation in each dimension. The crosses mark the crossover between the expression of the fidelity corresponding to the second and the third lines of Eq. (35). One notes that only for $d \geqslant 7$ there are values of the fidelity for which the entanglement of formation has to be evaluated according to the third line of Eq. (35).

In order to visualize how the entanglement itself is cloned, we plot in Fig. 5 the entanglement of formation $E_{F}$ of the clones as a function of the entanglement of formation of the input ME state $E_{\mathrm{in}}$, which is simply the von Neumann entropy of the reduced density matrix $E_{\text {in }}=\log _{2} d$. We note that the entanglement of the clones is always less than one half of the entanglement of the input state, while it asymptotically approaches this value for large $d$. The apparent «discontinuity» (if one can say so for a discrete graph) corresponds to $d=7$, i.e. the crossover between the second and the third lines of Eq. (35) when calculating the entropy of formation. In the limit of $d \rightarrow \infty$, the third line of Eq. (35) tends to $E_{F}=F \log _{2} d=F E_{\text {in }}$. Since the cloner can be viewed in this limit, as a classical random distribution process associated with a fidelity $F=1 / 2$, then the entanglement of each clone tends to one half of the entanglement of the initial state $E_{F} \rightarrow E_{\text {in }} / 2$. 
204 Karpov E., Navez P., Cerf N.J.

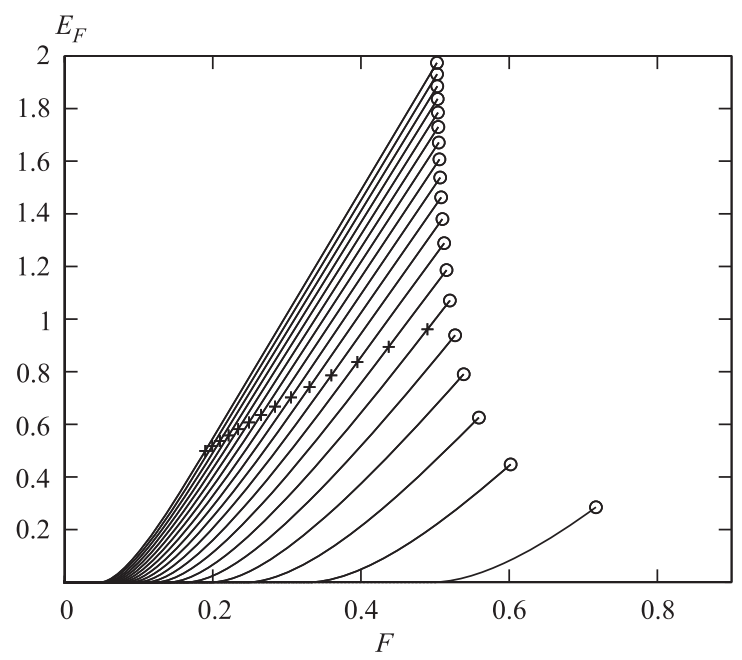

Fig. 4. Entanglement of formation $E_{F}$ of the clone of a maximally-entangled input state versus the fidelity $F$ of the clone for various dimensions $d=2-20$ (the lowest curve corresponds to $d=2$, while the highest curve corresponds to $d=20$ ). The circles show the maximum achievable fidelity and the corresponding entanglement of formation. The crosses mark the crossover between the expression of the fidelity corresponding to the second and the third lines of Eq. (35)

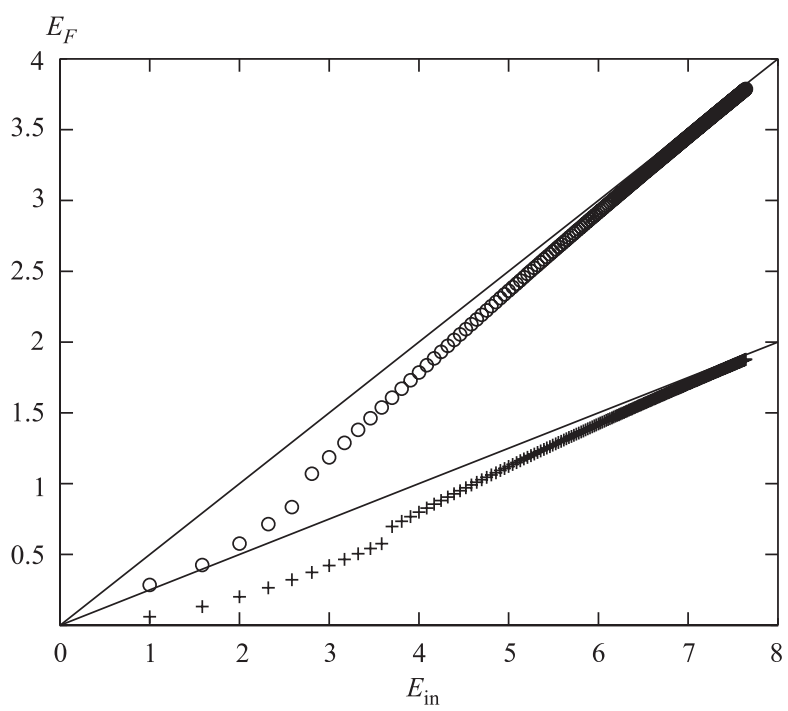

Fig. 5. Entanglement of formation $E_{F}$ of the clones of a maximally-entangled state obtained by the optimal (non-local) cloner (o) and the local cloner (+) versus the entanglement of the input state $E_{\text {in }}$ for various dimensions $d=2-200$. The apparent «discontinuity» in both curves is due to the crossover from the second to the third lines of Eq. (35) for $d \geqslant 7$ (optimal cloner) and $d \geqslant 13$ (local cloner). Solid lines represent the asymptotics of $E_{F}$ for large $d$ in both cases 
In Fig. 5, we also plot the entanglement of formation resulting from the «local» cloner discussed above. Recall that this cloner differs from our optimal (non-local) entanglement cloner only by setting $A=B$. Therefore, it is also covariant, satisfying Eq. (7), and all our arguments about the entanglement of formation of the clones are applicable to this cloner as well. Thus, using the fidelity of the clones (32), we may plot the entanglement of formation of the clones. We see that the local cloner leads to a lower entanglement of formation, and even the asymptotics of $E_{F}$ for large $d$ is no more than one fourth the entanglement of the input state. The reason is that in the limit of large $d$, the classical random distribution only succeeds with probability $1 / 2$ independently for Alice's and Bob's components, so the fidelity is $1 / 4$. Hence, $E_{F} \rightarrow E_{\text {in }} / 4$. These observations confirm that by increasing the dimensionality, we make the system behavior look more and more classical.

\section{SEPARABILITY CONSERVATION}

The last point to check is that our cloner does not create entanglement by itself, that is, it clones separable states into separable states. First, an important observation is that our cloner is such that the input-to-single-clone transformation is a positive partial transpose (PPT) map. Using Eqs. (5) and (10), and tracing the joint state $|S\rangle_{\mathcal{R}, a, b, \mathcal{A}}$ over the ancilla $\mathcal{A}$ and one of the clones, say $b$, we arrive at the following expression for the state of the reference and the other clone

$$
\begin{aligned}
S_{\mathcal{R}, a}= & |A|^{2}\left(\mathbb{1}_{A} \otimes \mathbb{1}_{B}\right)_{\mathcal{R}, a}+ \\
& +d^{2}\left(\left(d^{2}+2\right)|A|^{2}+2|B|^{2}+4 d \operatorname{Re}\left(A^{*} B\right)\right)\left(|\phi\rangle_{A}\left\langle\left.\phi\right|_{A} \otimes \mid \phi\right\rangle_{B}\left\langle\left.\phi\right|_{B}\right)_{\mathcal{R}, a}+\right. \\
& +d\left(d|B|^{2}+2 \operatorname{Re}\left(A^{*} B\right)\right)\left(|\phi\rangle_{A}\left\langle\left.\phi\right|_{A} \otimes \mathbb{1}_{B}+\mathbb{1}_{A} \otimes \mid \phi\right\rangle_{B}\left\langle\left.\phi\right|_{B}\right)_{\mathcal{R}, a},\right.
\end{aligned}
$$

where $\mathbb{1}_{A}$ is the identity operator in the joint space of Alice's component of the reference $\mathcal{R}$ and clone $a$, while $|\phi\rangle_{A}=d^{-1 / 2} \sum_{i=0}^{d-1}|i\rangle_{\mathcal{R}_{A}}|i\rangle_{a_{A}}$ is an ME state in the same space. (The same notations are used for Bob's analog quantities $\mathbb{1}_{B}$ and $|\phi\rangle_{B}$.) The cloning map is thus PPT since $\left(S_{\mathcal{R}, a}\right)^{T_{B}} \geqslant 0$, where $T_{B}$ stands for the partial transposition with respect to Bob's components of the reference $\mathcal{R}$ and clone $a$. This PPT property ensures that the cloning of any isotropic state cannot increase its fidelity, hence its entanglement of formation [15]. In particular, all separable isotropic states are necessarily transformed into separable clones.

In order to generalize this separability conservation property to all separable input states outside the restricted class of isotropic states, we consider the cloning of a product state $\rho_{A} \otimes \rho_{B}$. By tracing $\left(\rho_{A} \otimes \rho_{B}\right)^{T} S_{\mathcal{R}, a}$ over the reference $\mathcal{R}$, we obtain for the first clone a state of the form

$$
\begin{aligned}
\rho_{a}=|A|^{2}\left(\mathbf{1}_{A} \otimes \mathbb{1}_{B}\right)_{a}+\left(\left(d^{2}+2\right)|A|^{2}+2|B|^{2}+4 d \operatorname{Re}\left(A^{*} B\right)\right)\left(\rho_{A} \otimes \rho_{B}\right)_{a}+ \\
+\left(d|B|^{2}+2 \operatorname{Re}\left(A^{*} B\right)\right)\left(\rho_{A} \otimes \mathbb{1}_{B}+\mathbb{1}_{A} \otimes \rho_{B}\right)_{a},
\end{aligned}
$$

where $\mathbb{1}_{A}$ and $\mathbb{1}_{B}$ are identities in Alice's and Bob's subspaces of clone $a$, respectively. Since all terms in (40) are product states and all coefficients are positive semi-definite for all $d$, we verify that $\rho_{a}$ is separable. By linearity of the trace, this result also holds for any linear 
combination $\sum_{i} p_{i} \rho_{i}^{A} \otimes \rho_{i}^{B}$ with $p_{i} \geqslant 0$ and $\sum_{i} p_{i}=1$, that is, for a generic separable state. Thus, we can conclude that our entanglement cloner transforms all initially separable states into separable clones.

\section{CONCLUSION}

In conclusion, we have constructed an optimal (symmetric) entanglement cloner, which is universal over the set of $d \times d$ ME states. On the one hand, all separable input states are cloned by this cloner into separable states. On the other hand, the entanglement of the clones of $\mathrm{ME}$ input states is maximum. In addition, the entanglement of isotropic states cannot be increased by the cloner (and we conjecture that this property holds in general for any input state).

The optimization of the parameters of our QCM was performed by maximizing the fidelity of the clones, but the monotonic behavior of the entanglement of formation as a function of the fidelity for isotropic states guarantees that such an optimization maximizes the entanglement of the clones at the same time. We expect that entanglement is cloned «monotonically» also for non-isotropic states, that is, higher entangled states result in higher entangled clones, and therefore the ME input states are those which generate the clones with the maximum achievable entanglement. If this very natural assumption is right, then, based on our result, one can state that our cloner optimally duplicates the entanglement of any pair of $d$-dimensional quantum system. Moreover, the maximal entanglement attainable by cloning is always below one half of the entanglement of the input state and saturates this value in the limit of large dimension $d$. This is consistent with the idea that, since our QCM transforms separable states into separable clones, no additional entanglement is produced by cloning, so we can only split the entanglement of the input state between the two clones. This explains as well the asymptotic value of one fourth the initial entanglement for the local cloner at the limit of large $d$. It is natural to expect that all these conclusions remain valid for asymmetric entanglement cloners as well.

Acknowledgements. We thank Louis-Philippe Lamoureux and Jaromir Fiurasek for helpful discussions. We acknowledge the support from the European Union under the projects RESQ (Grant No. IST-2001-37559) and SECOCQ (Grant No. IST-2002-506813), from the Communauté Française de la Belgique under the «Action de Recherche Concertée» nr. 00/05251, and from IAP program of Belgian federal government under Grant No. V-18. P. Navez aknowledges support from Junior Fellowship F/05/011 of the KULeuven Research Council.

\section{REFERENCES}

1. Karpov E., Navez P., Cerf N.J. Cloning Quantum Entanglement in Arbitrary Dimensions // Phys. Rev. A. 2005 (in press).

2. Wooters W. K., Zurek W. H. // Nature (London). 1982. V.299. P. 802; Dieks D. // Phys. Lett. 1982. V.92A. P. 271.

3. Bužek V., Hillery M. Quantum Copying: Beyond the No-cloning Theorem // Phys. Rev. A. 1996. V. 54. P. 1844 
4. Lamoureux L.-Ph. et al. Cloning the Entanglement of a Pair of Quantum Bits // Phys. Rev. A. 2004. V. 69. P. 040301(R).

5. Cerf N. J. Special Issue on Quantum Information // Acta Phys. Slov. 1998. V. 48. P. 1115;

Cerf N. J. Asymmetric Quantum Cloning in Any Dimension // J. Mod. Opt. 2000. V.47. P. 187; Cerf N. J. Pauli Cloning of a Quantum Bit // Phys. Rev. Lett. 2000. V. 84. P. 4497.

6. Jamiolkowski A. // Rep. Math. Phys. 1972. V.3. P. 275.

7. Fiurášek J. Extremal Equation for Optimal Completely Positive Maps // Phys. Rev. A. 2001. V.64. P. 062310 .

8. Dieulesaint I., Royer D. Group Theory and Its Application to Physical Problems / Transl. by A. Bastin, M. Motz. Chichester; N. Y.; Brisbane; Toronto: Wiley-Intersci., John Wiley \& Sons, 1980. $141 \mathrm{p}$.

9. Navez P., Cerf N. J. Cloning a Real $d$-dimensional Quantum State on the Edge of the No-Signalling Condition // Phys. Rev. A. 2003. V. 68. P. 032313.

10. Demkowicz-Dobrzański R. et al. Classical Communication May Improve Local Cloning of Entangled Quantum States. quant-ph/0502103. 2005. P.4.

11. Bennett C. H. et al. Mixed-State Entanglement and Quantum Error Correction // Phys. Rev. A. 1996. V.54. P. 3824.

12. Vollbrecht K. G.H., Werner R. F. Entanglement Measure under Symmetry // Phys. Rev. A. 2001. V.64. P. 062307.

13. Horodecki M., Horodecki P. Reduction Criterion of Separability and Limits for a Class of Distillation Protocols // Phys. Rev. A. 1999. V.59. P. 4206.

14. Terhal B. M., Vollbrecht K. G. H. Entanglement of Formation for Isotropic States // Phys. Rev. Lett. 2000. V.85. P. 2625.

15. Rains E. M. A Semidefinite Program for Distillable Entanglement // IEEE Trans. Inf. Theory. 2001. V. 47. P. 2921 